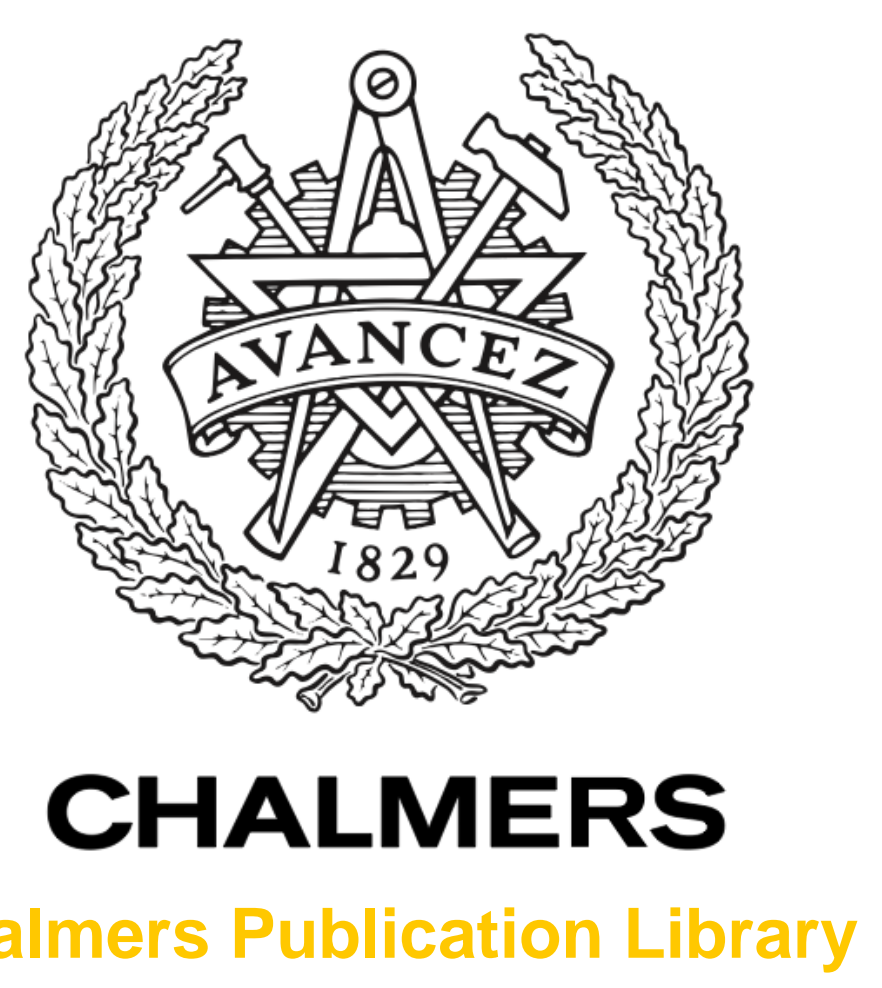

Chalmers Publication Library

\author{
Evaluation of collision avoidance prototype head-up display interface for older \\ drivers
}

This document has been downloaded from Chalmers Publication Library (CPL). It is the author's version of a work that was accepted for publication in:

Lecture Notes in Computer Science. 14th International Conference on Human-Computer Interaction, HCl International 2011, Orlando, 9-14 July 2011 (ISSN: 0302-9743)

Citation for the published paper:

Charissis, V. ; Papanastasiou, S. ; Mackenzie, L. (2011) "Evaluation of collision avoidance prototype head-up display interface for older drivers". Lecture Notes in Computer Science. 14th International Conference on Human-Computer Interaction, HCI International 2011, Orlando, 9-14 July 2011, vol. 6763 pp. 367-375.

http://dx.doi.org/10.1007/978-3-642-21616-9_41

Downloaded from: http://publications.lib.chalmers.se/publication/154869

Notice: Changes introduced as a result of publishing processes such as copy-editing and formatting may not be reflected in this document. For a definitive version of this work, please refer to the published source. Please note that access to the published version might require a subscription.

Chalmers Publication Library (CPL) offers the possibility of retrieving research publications produced at Chalmers University of Technology. It covers all types of publications: articles, dissertations, licentiate theses, masters theses, conference papers, reports etc. Since 2006 it is the official tool for Chalmers official publication statistics. To ensure that Chalmers research results are disseminated as widely as possible, an Open Access Policy has been adopted.

The CPL service is administrated and maintained by Chalmers Library. 


\title{
Evaluation of Collision Avoidance Prototype Head-Up Display Interface for Older Drivers
}

\author{
Vassilis Charissis ${ }^{1}$, Stylianos Papanastasiou ${ }^{2}$, Lewis Mackenzie ${ }^{3}$, and Sachi Arafat ${ }^{3}$ \\ ${ }^{1}$ Glasgow Caledonian University \\ School of Engineering and Computing, \\ Division of Computing and Creative Technologies, \\ Glasgow, UK \\ ${ }^{2}$ Chalmers Institute of Technology, \\ Department of Communication Systems and Information Technology, \\ Goteborg, Sweden \\ ${ }^{3}$ University of Glasgow, School of Computing, Glasgow, UK \\ v.charissis@gmail.com, stypap@chalmers.se, \\ \{lewis,sachi\}@dcs.gla.ac.uk
}

\begin{abstract}
Spatial and situational awareness could be decreased significantly under low visibility and adverse weather conditions. This could affect exponentially the reactions of the older drivers and increase dramatically their collision probability. To this end we developed a novel Head-Up Display interface that aims to reinstate the drivers' vision which is predominantly hindered under these conditions. In particular the proposed interface entails symbolic representations of the lead vehicles and crucial road information, which effectively enhances driver's vision. The proposed system was evaluated through a comparative study against the typical instrumentation panel. The evaluation results were overall in favour of the prototype interface which improved significantly the reaction times of the older drivers and decreased the collision occurrences.
\end{abstract}

Keywords: Older drivers, Head-Up Display, HCI, Driving Simulator, low visibility, navigation system, visual interface, collision avoidance.

\section{Introduction}

Perception and analysis of visual information whilst driving can be a daunting process, particularly for the older drivers, in whom spatial perception and response times tend to be reduced [1]. The latter category represents a rapidly growing segment of the drivers' population requiring additional support in order to counteract these physical and mental age related inefficiencies. Previous studies have demonstrated that the cognitive capabilities and senses deteriorate in geometric progression to age [2]. Interestingly, the produced vehicle interior systems offer a wealth of information and entertainment devices which can additionally hamper even the average drivers' attention.

As such, the over-elaborated, or over-simplified in some rare cases, design of invehicle interfaces do not take into consideration the physical and cognitive limitations

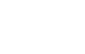


that older drivers present. In particular, the navigation systems present various road information that older drivers cannot interpret or use in a timely manner [3]. Recently, technological advances produced numerous collision-avoidance systems which have been tentatively and gradually incorporated into contemporary vehicles. Yet the vast majority alerts the driver before an imminent collision and relies heavily on the driver's response time. A small segment of experimental systems even retains the control of the vehicle and regulates the vehicular activities until it is safe to return the control to the driver. Yet both solutions do not provide a viable solution to the older drivers, which either cannot respond in time on the alerts, or they might feel completely intimidated by the temporarily autonomous navigation of their vehicle. Moreover, adverse weather conditions and unexpected traffic congestion can diminish even further older drivers' reaction times, increasing significantly the possibility of collision occurrence and fatalities.

Mindful of the aforementioned observations, our primary objective was to indicate to the user well in advance, potential collisions and subsequently improve the driver's responses without excluding him/her from the actual driving and decision making. To this end, we have developed a full-windshield, Head-Up Display interface, in order to provide the driver with essential, timely presented collision avoidance information [4]. The provided information is superimposed on the windshield through symbolic representation of the forthcoming obstacles and vehicles. The representations are mainly based on the human ability to identify an object based solely on the bordershape of it [1]. Additionally we introduced patterned design colour and size coding in order to intonate the significance of each symbol. Our initial evaluation trials were encouraging, showcasing significantly improved response times and decreased collision occurrences. Yet they provided an insight to previously unforeseen issues that prevented the particular group to capitalize fully the offered information. Through an incremental process of interface design and functionality improvements we managed to incorporate the majority of the older driver's suggestions into the final system. In particular this paper presents the design process of a prototype Head-Up Display (HUD) interface, which aims to improve the driver's spatial awareness and response times under low visibility conditions. The working interface prototype has been further improved for older drivers' use.

Interestingly our previous work towards the development of an all-inclusive users' HUD interface has indicated that such interfaces may enhance significantly the driver's spatial and situational awareness resulting in faster responses and successful collision avoidance [4].

The paper discusses in detail the interface design mantra and presents the results of a large scale comparative evaluation of the HUD system versus the typical dashboard (HDD) information on a group of forty users. Furthermore this work presents an exhaustive analysis of users' age, gender, collision occurrences and the collision speed $(\mathrm{m} / \mathrm{sec})$ of each user, which can offer an informative appraisal of the effectiveness of the HUD system through the estimation of collisions per trial, with and without the HUD interface. Concluding, this work describes the HUD interface development towards a user-friendly system which could enhance the response times of the older users. 


\section{Visual and Cognitive Issues of Older Drivers}

The driving process involves multiple senses and cognitive functions and requires precise coupling of mind and body reactions [5].

In particular, visual sensory functions that decline with age are namely; the visual acuity, useful field of vision (UFOV), sensitivity to glare, contrast sensitivity and colour perception [6].

In turn spatial and situational awareness can be detrimentally affected, jeopardising the safety of the older driver [7]. The decline of cognitive and visual sensory abilities challenges the driving performance of older users $[8,9]$. More analytically the visual acuity which allows the driver to see objects in detail from a distance decreases gradually after the age of 20 y.o., and declines rapidly after the age of 50 y.o. [10, 11]. Notably this exponential decline of visual acuity is not perceivable by the majority of the elderly driving population [6].

A previous study identified a clear relation between decreased visual acuity and crash rates of older drivers [13]. Older users' vision is further affected by the gradual limitation of the useful field of view, which is the area of the visual field that accommodates information quickly perceivable without any eye or head movements [6]. The significantly reduced visual estate reduces the older drivers' ability to identify objects or movement on the extreme sides of their visual frame, such as vehicles in adjacent lanes [6,7]. The vision sense deterioration of the older users is also affected by the declining ability to distinguish colours [12]. Hence colour perception diminishes significantly up to $50 \%$ in the age of 90 y.o [13]. As such the older drivers require clear schematic information which does not rely solely on the colour differentiation between the various states of the information.

Finally the cognitive functions are also affected by age, preventing older drivers to obtain, store and use information [13, 14]. Decreased cognitive ability has an imminent negative impact to the response times (RTs) of older drivers which struggle to make critical decisions under pressure $[5,8]$.

\section{HUD Interface}

The aforementioned observations and facts suggested that a novel vehicular navigation system should compensate for the visual impairment of older users and enhance their response time to critical situations without overloading their cognitive functions. As such we strived towards the development of a modular interface which could be accommodated in the direct field of view of the user through the form of a Head-Up Display (HUD). HUD systems have been predominantly used in military aircrafts as they inherently increase screen estate for the real-time presentation of a multitudinous flight and combat information. In tentative steps, HUD has been introduced in the automotive and marine transportation. In particular the early automotive applications offered a new field of experimentation and presentation of additional information collated to the actual driving environment. Concurrent versions investigate the interface conduit which will provide supplementary driving-related information, thus complementing the traditional dashboard, while attracting attention as it is positioned within the driver's immediate field of view. 
To this end we have developed a simple interface design which may provide crucial information excluding content that is already presented on the congested dashboard and alert driver for the potential collision risks. The proposed humancomputer interface deployed on a full-windshield HUD system, aims to reinstate the three main cognitive factors that affect the older drivers' responses, namely: visual processing speed, ability to divide attention and target clarity [7].

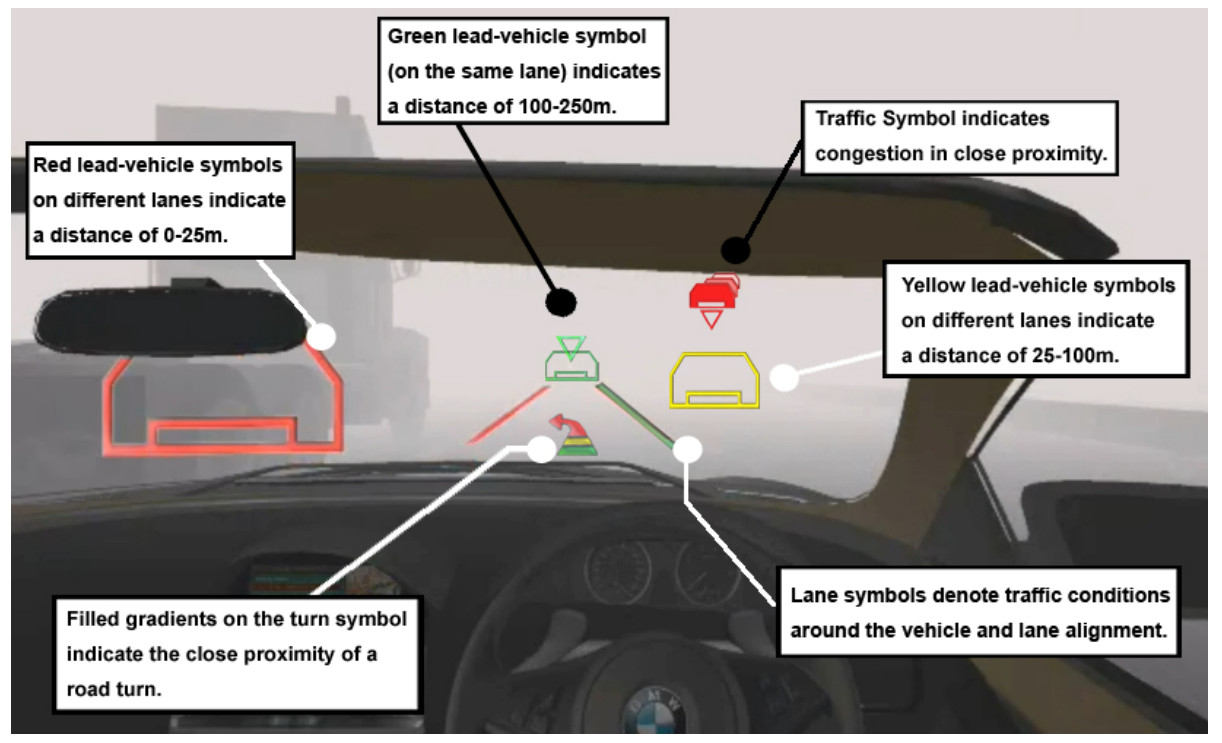

Fig. 1. Screenshot of the actual Driving and brief HUD interface explanatory information

The acquisition and interpretation of a plethora of information available through vehicular sensors, enables the HUD interface to convey this information to the user in an uncluttered and simplified visual output. As such the system could enhance the perception of the vehicle's surrounding space and improve the driver's response times, particularly under low visibility conditions. Our initial experimentations and the subsequent evaluations, informed further our interface design and functionality regarding the fine tuning of the quantity, quality, timing and presentation of the visual cues.

In tandem, the system offers symbolic representation of the vehicles and surroundings of a motorway environment, such as lead vehicles, lane recognition, sharp turn warning and traffic alert. These symbols trace the actual vehicles, lane positioning and curvature and embed themselves to the environment through the Head-Up Display projection system as illustrated in Figure 1. Their size and colour-coding alters respectively with the distance and time to collision (TTS) parameters provided by the vehicular ad-hoc network system (VANETS) that gathers this surrounding information. 


\section{Modelling and Simulation}

In order to evaluate the improved prototype interface, a custom driving simulator was developed to investigate the efficiency of a full-windshield Head-Up Display (HUD) interface for the elder drivers' in low visibility and traffic congestion conditions. The driving-simulator (OSDS) was based on an open source code and off-the-shelf hardware components [4].

The numerical information acquired during the experiment traced primarily the actual response times (RT) and headway (HW) benefits derived with and without the HUD usage. However being aware of the plethora and complexity of factors involved in an imminent collision such as visibility, psychological refractory period, spatial awareness, situational awareness and stimulus-response compatibility amongst others, we employed various methods of recording the human responses such as heart-rate, eye-tracking, body posture and facial expressions.

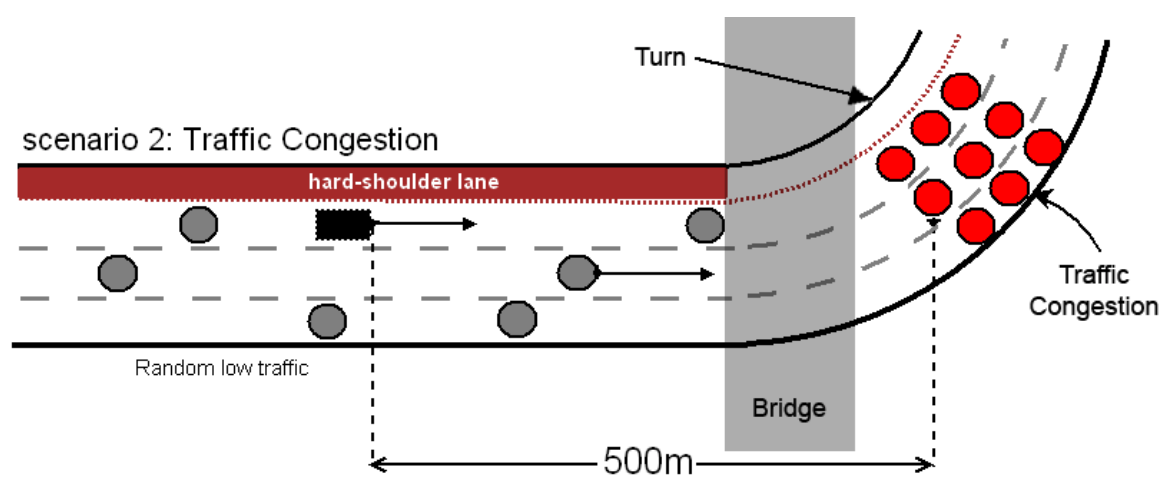

Fig. 2. Traffic congestion and sharp turn situations (second scenario)

The simulation scenario entails low visibility, sharp turn and traffic congestion practically invisible positioned behind a bridge maximizing the effect of surprise as depicted in Figure 2. In addition the agent vehicles of the normal flow and the traffic congestion were infused with a degree of artificial intelligence in order to increase the realism of the simulation scenarios [15]. Their individual driving patterns embedded on the agent vehicles were derived from real-life observations and driver behaviours defined by proprietary literature. The different accident scenarios were kindly provided by the Strathclyde Police Department in Glasgow.

Notably in this scenario the drivers performed adequately in comparison to other simulated scenarios. Yet the collisions occurrences were still at alarming levels.

In accordance to our previous evaluation methods the second scenario trials attracted 40 volunteer drivers with valid driving licence and aging between 20 and 75 years old. The drivers were randomly selected in order to represent the largest possible part of driving population spanning across a large variation of driving experience, profession, gender and age. 
The primary aim of the experiment's design was to evaluate the benefits, if any, of using the HUD during driving under low visibility conditions in a motorway environment with particular interest to improve significantly the response times of the elder users.

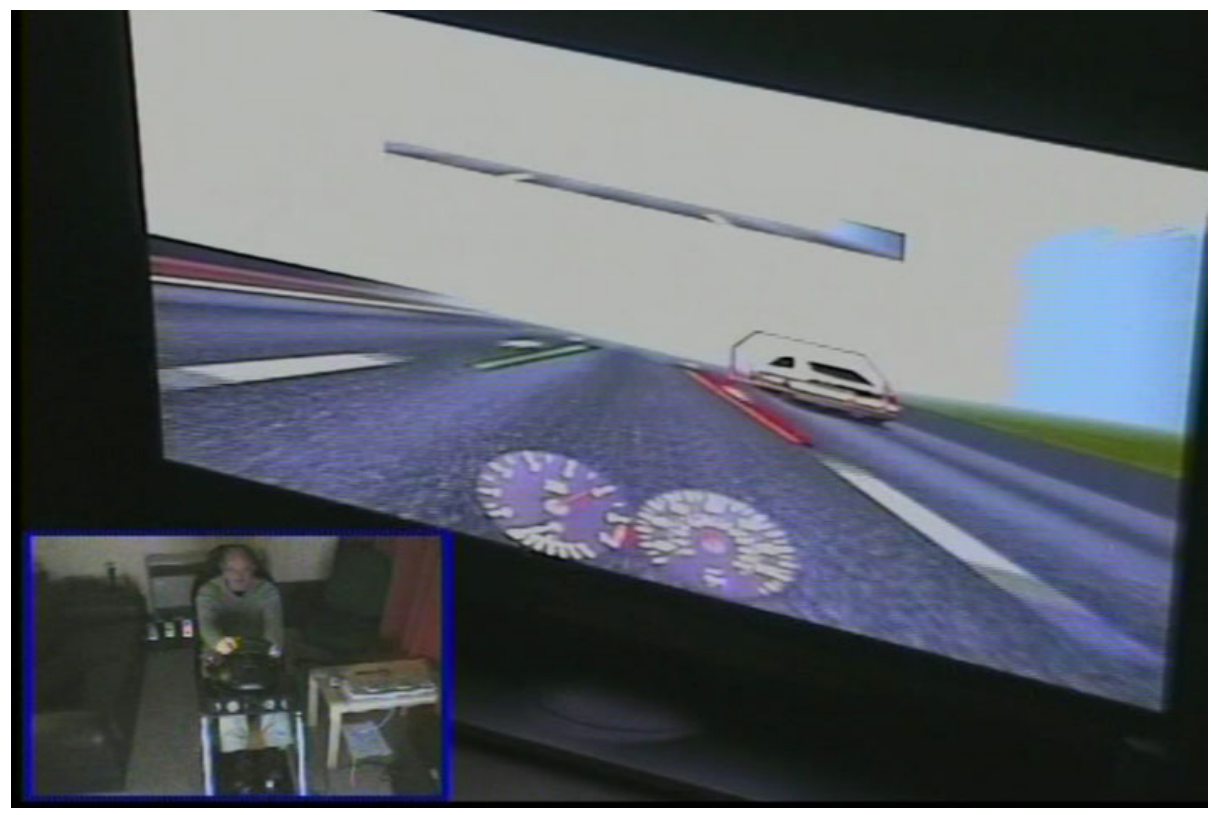

Fig. 3. Screenshot of the user video footage and the driving simulator in action

\section{Results and Discussion}

The analysis of the numerical data offered a precise view of the drivers' reactions, benefits and drawbacks of the proposed HUD interface in contrast to the existing HDD system. Yet further observation of the video footage and the subjective feedback verifies the numeric information and highlights the overall user experience during the different stages of the experiment. In particular, the video-captures presented distinguishable body-postures during the simulation of the accident scenarios with and without the HUD system.

Further observations highlighted that the drivers without the use of HUD support, tended to keep their elbows closer to the body, hold tight the steering wheel and in some cases, leaning forward on the driving seat. Their anxiety was also evident through their facial expressions. During the subjective feedback and interview they noted that the low-visibility due to the simulated fog produced a very difficult and dangerous driving environment which was very uncomfortable and distressing.

Interestingly, the older drivers recognised the symbols' functionalities and familiarised themselves with the HUD interface within minutes. The users commented positively on the simplicity of the system and the unusual comfort of not needing 
prolix manual instructions, typically accompanying the majority of navigation systems and other infotainment devices. This was a common observation made by all the forty drivers that participated in the experiment. Furthermore the users commented positively on the full-windshield projection, which immersed the highlighted symbols with the actual scenery, enabling the older users to avoid imminent collisions.

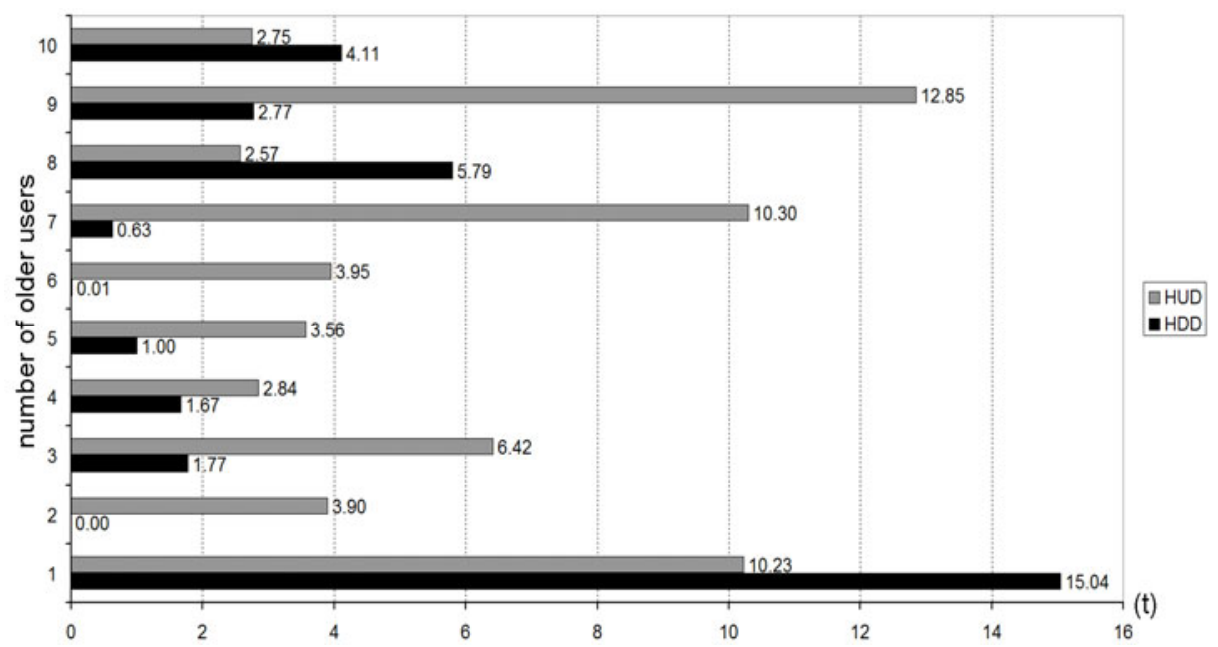

Fig. 4. Comparison of collision speeds (a) with HUD and (b) with HDD

The subjective feedback was also supported form the numerical findings. Focusing particularly on the older drivers group, the effect of the HUD usage became immediately visible as the simulation recorded significantly decreased collision occurrences.

An analysis of their driving behaviour through the numerical data revealed that the older users managed to maintain a sufficient time-to-collision (TTC) window with the lead vehicle/obstacle with the use of the HUD interface. In contrast the use of HDD did not offer sufficient information which resulted in a significantly minute TTCs and increased collisions as presented in Figure 4.

Notably users 4, 7 and 10 although maintained their distance from the lead vehicles they lost the control of their vehicle in the road-curve negotiation, although they managed to maintain their distance from the lead vehicles and the traffic congestion. As such $50 \%$ of the older drivers that participated in this trial collided either with the vehicles forming the traffic bottleneck or with the difficult hairpin type of road curve.

In contrast none of the drivers collided with these simulation elements when the HUD interface was activated. Another interesting finding was the follow-up speed of the lead vehicles that the older users maintained which was constantly higher with the use of the proposed interface as depicted in Figure 5, without though exceeding the motorway limits.

This can be attributed to the fact that the highlighted information by the HUD interface offered a significant advantage to the drivers and particularly to the older population by enhancing effectively their vision. In turn the users could trace the traffic and the indistinguishable objects more than $250 \mathrm{~m}$ ahead even in low visibility 


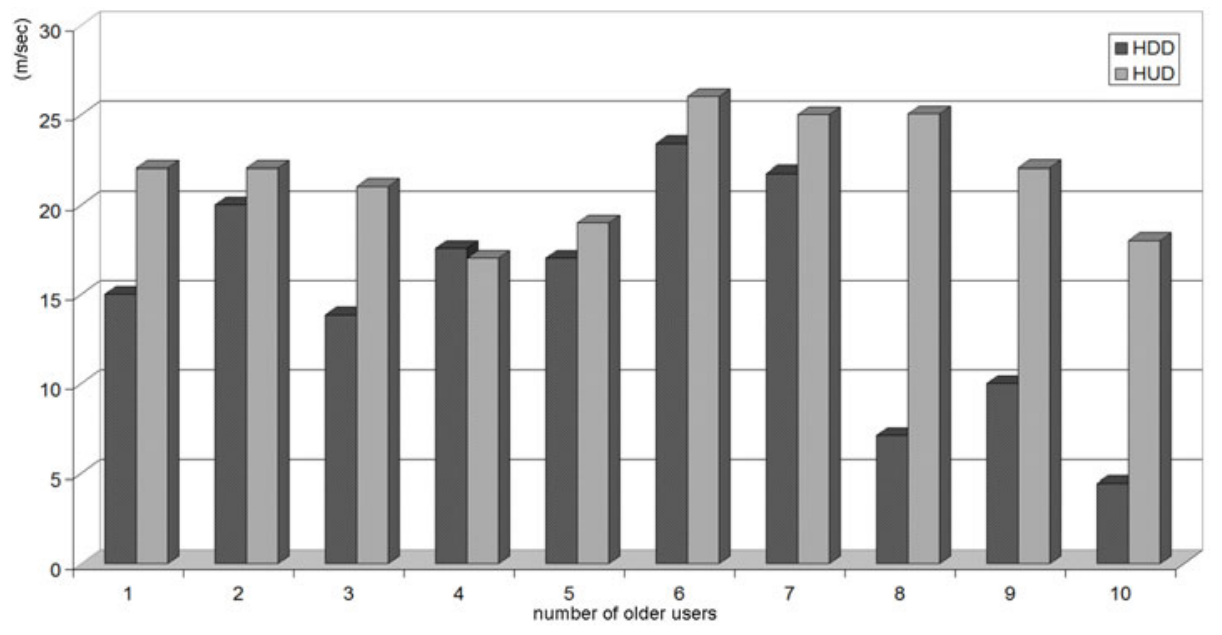

Fig. 5. Comparison of follow-up speeds (a) with HDD and (b) with HUD

conditions. As a result the drivers could produce the typical motorway speeds, yet remaining calm and in control of their vehicle.

\section{Conclusions}

This paper offered a succinct presentation of the contemporary issues hindering the older driving population. In turn, we presented our proposed guidance system which entails a prototype HUD interface deployed in full-windshield. Adhering to the typical spatial and situational awareness issues of the older drivers the system provides the users with simplified visual information, yet crucial for the effective collision avoidance. The evaluation of the system against the contemporary instrumentation panels, suggests that the proposed HUD improves significantly the users' reactions and effectively decreases the collision occurrences. However the system does not currently offer any customisation attributes. As the majority of the drivers increased substantially their speed the system should be enabled in the future to advise the user for the optimal driving speed in order to avoid abuse of the system's benefits.

Finally our future plan is the incorporation of auditory and haptic cues which will complement the visual HUD warnings. Furthermore we envisage the development of an automated calibration system which will optimise the visual HUD inputs according to the visual characteristics of each user.

\section{References}

1. Hoffman, D.D., Richards, W.A.: Parts of Recognition. In: Pinker, S. (ed.) Visual Cognition, pp. 65-96. MIT Press, Cambridge (1985)

2. Rakotonirainy, A., Steinhardt, D.: In-vehicle technology functional requirements for older drivers. In: Proceedings of the 1st International Conference on Automotive User Interfaces and Interactive Vehicular Applications, Essen, Germany, pp. 27-33 (2009) ISBN:978-160558-571-0 
3. Itoh, N., Yamashita, A., Kawakami, M.: Effects of car-navigation display positioning on older drivers' visual search, in Assessment and Promotion of Work Ability, Health and Well-being of Ageing Workers. Journal of International Congress Series 1280, 184-189 (2005)

4. Charissis, V., Vlachos, G., Chan, W., Arafat, S.: Early Notification Warning System for Prototype Head-Up Display: Development and Evaluation of Traffic Congestion and Sharp Turn Warnings. In: Proceedings of the Society of Automotive Engineers (SAE) World Congress, Detroit, Michigan, USA, April, 12-15 (2010)

5. Musselwhite, C.B.A., Haddad, H.: Exploring older drivers' perceptions of driving. European Journal of Ageing 7(3), 181-188 (2010)

6. European Road Safety Observatory (2006) Older Drivers, http://www.dacotaproject.eu/Links/erso/knowledge/Fixed/07_old/olderdrivers.pdf (retrieved, November 10, 2010)

7. Ball, K.: Attentional Problems and Older Drivers. Alzheimer Disease and Associated Disorders 11(suppl. 1), 42-47 (1997)

8. Clarke, D.D., Ward, P., Bartle, C., Truman, W.: Older drivers' road traffic crashes in the UK. Accident Analysis Prevention 42(4), 1018-1024 (2010)

9. Ryu, J., Song, G., Lee, S., Cho, Y., Kyung, G., Kim, H., Baek, K.: Improving in-Vehicle Display and Control Design for Older Drivers. In: Karsh, B.-T. (ed.) EHAWC 2009. LNCS, vol. 5624, pp. 170-176. Springer, Heidelberg (2009)

10. Meyer, J.: In-Vehicle Telematics Systems and the Older Driver. The Silver Market Phenomenon 2008, IV, pp. 381-390 (2008)

11. Owsley, C., McGwin Jr., G.: Vision and Driving. Vision Res. 50(23), 2348-2361 (2010)

12. Eby, D.W., Trombley, D., Molnar, L.J., Shope, J.T.: The Assessment of older driver's capabilities: A review of the literature (Report No. UMTRI-98-24). University of Michigan Transportation Research Institute, Ann Arbor, MI (1998)

13. Bayam, E., Liebowitz, J., Agresti, W.: Older drivers and accidents: A meta and data mining application on traffic accident data. Expert Systems with Applications 29, 598-629 (2005)

14. Caird, J.K., Chisholm, S.L., Lockhart, J.: Do in-vehicle advanced signs enhance older and younger drivers' intersection performance? Driving simulation and eye movement results. International Journal of Human-Computer Studies 66(3), 132-144 (2008)

15. Charissis, V., Papanasasiou, S.: Human-machine collaboration through vehicle head up display Cognition. Technology \& Work 12(1), 41-50 (2008) 